\title{
Molecular tailoring approach for geometry optimization of large molecules: Energy evaluation and parallelization strategies
}

\author{
V. Ganesh, ${ }^{\text {a) }}$ Rameshwar K. Dongare, P. Balanarayan, and Shridhar R. Gadre ${ }^{\text {b) }}$ \\ Department of Chemistry, University of Pune, Pune 411007, India
}

(Received 10 April 2006; accepted 31 July 2006; published online 13 September 2006)

\begin{abstract}
A linear-scaling scheme for estimating the electronic energy, gradients, and Hessian of a large molecule at $a b$ initio level of theory based on fragment set cardinality is presented. With this proposition, a general, cardinality-guided molecular tailoring approach (CG-MTA) for ab initio geometry optimization of large molecules is implemented. The method employs energy gradients extracted from fragment wave functions, enabling computations otherwise impractical on PC hardware. Further, the method is readily amenable to large scale coarse-grain parallelization with minimal communication among nodes, resulting in a near-linear speedup. CG-MTA is applied for density-functional-theory-based geometry optimization of a variety of molecules including $\alpha$-tocopherol, taxol, $\gamma$-cyclodextrin, and two conformations of polyglycine. In the tests performed, energy and gradient estimates obtained from CG-MTA during optimization runs show an excellent agreement with those obtained from actual computation. Accuracy of the Hessian obtained employing CG-MTA provides good hope for the application of Hessian-based geometry optimization to large molecules. (C) 2006 American Institute of Physics. [DOI: 10.1063/1.2339019]
\end{abstract}

\section{INTRODUCTION}

The computational power and storage have been driven by Moore's law ${ }^{1}$ in the past few decades, and would probably continue to do so for a few more decades. With readily available processing power, a computational scientist can routinely apply $a b$ initio theories for attempting solutions of many challenging problems in chemistry, physics, and biology. However, the formidable computational complexity of these methods ${ }^{2-5}$ is a major bottleneck towards applying them to larger chemical or biological systems. For a system with $N$ basis functions, though the complexity is theoretically $O\left(N^{4}\right)$ for the Hartree-Fock (HF) method [and going up to $O\left(N^{7}\right)$ for correlated methods], it has been well known that only $O\left(N^{2}\right)$ integrals are significant for a very large molecule. ${ }^{6}$ In consequence, even with huge computational resources, practical applications of conventionally coded $a b$ initio methods are plausible only for the systems containing fewer than 100 atoms at a sufficiently reliable level of theory and basis.

In an attempt to reduce the CPU and memory requirements of $a b$ initio methods, linear-scaling divide-andconquer (DC)-type algorithms have had a fair amount of success in computing one-electron properties and in structure determination. ${ }^{7-21}$ Yang and Lee ${ }^{8}$ reported one of the earliest attempts in applying DC-type methodology for geometry optimization with density functional theory (DFT). Although the results obtained by them were reported to be close to the actual computations (when applied to small molecules like a $\beta$-tetrapeptide comprising of glycine), there was no apparent advantage in terms of CPU time when compared to the cor-

\footnotetext{
a) Also at the Interdisciplinary School of Scientific Computing, University of Pune.

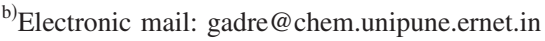

responding full Kohn-Sham calculation. Exner and Mezey ${ }^{16}$ have developed and employed their adjustable density matrix approximation (ADMA) to various classes of polypeptides for determining one-electron properties. Using molecular fractionation with conjugate caps (MFCC) approach, developed by $\mathrm{Li}$ et $a l^{18}$ and later extended by Chen et al., ${ }^{22}$ the estimations of HF, Møller-Plesset second order perturbation (MP2), and B3LYP energies with maximum error of up to a few millihartrees have been demonstrated. However, most of the systems reported by the previous researchers are either small or linear except for the case of crambin molecule. ${ }^{23}$ Although the geometry optimization of crambin molecule appears to be one of the largest $a b$ initio calculation being carried out so far, we find that there are still sufficient computational bottlenecks that need to be overcome for a routine application of these methods to biologically interesting systems. In the previous works, ${ }^{11-15}$ our group has independently developed a molecular tailoring approach (MTA) and has applied it for the computation of one-electron properties such as electron density and electrostatic potential of many large nonlinear molecules such as silicalite, ibuprofen cluster, and many others. As an initial attempt towards applying MTA for geometry optimization, a small albumin binding protein of 851 atoms was partially optimized at the HF/6$31 \mathrm{G}$ level using relatively inexpensive and distributed PC resources. ${ }^{15}$

In an almost parallel, independent development, Kitaura's group have developed a similar fragment-based strategy termed fragment molecular orbital (FMO) method which has been incorporated into the GAMESS package. ${ }^{19,20,24-27}$ Komeiji et al. have also applied FMO to molecular dynamics simulations. ${ }^{28}$ However, the FMO technique has been largely tested and applied for single point energy evaluation of large molecules, obtaining binding/interaction energies of protein 
or protein-ligand complexes, ${ }^{24,26}$ etc. Single point calculation on lysozyme, a protein with about 2000 atoms at HF/4-31G level has also been reported using the FMO method by Nakano et al. ${ }^{19}$ and Kitaura et $a l^{20}$ A computational time of four days with a cluster of 18 Pentium III computers was reported for this investigation. The largest of the FMO enabled calculation, which has recently been reported ${ }^{24}$ is on 20881 atom photosynthetic protein at RHF/6-31G(d) level of theory using a huge computing power of $600 \mathrm{CPUs}$. A few systems have also been subjected to geometry optimization, predominantly within the FMO-HF framework. On the contrary, MTA scheme [presented here as cardinality-guided MTA (CG-MTA)], apart from being used for geometry optimization (to obtain electronic energy and its derivatives), has earlier been shown to be useful for obtaining the density matrix $^{12-15}$ and one-electron properties of molecule. The CGMTA scheme is now being applied to geometry optimization at HF, B3LYP, and MP2 level theories. We present detailed comparisons with the actual run as well as sequential and parallelization benchmarks against actual geometry optimization run. The default parallelization scheme used in CGMTA is designed for a loosely connected heterogeneous clusters of PCs and is described later in Sec. II.

There has also been growing interest in developing methods for geometry optimization of large molecules. Of these, the ONIOM (our own $n$-layered integrated molecular orbital and molecular mechanics) (Refs. 29-31) method, which is available through a popular $a b$ initio package, ${ }^{29}$ GAUSSIAN 03, has been extensively used in recent years. These "semi" ab initio methods usually apply a lower level of theory in the outer region of the molecule and a sufficiently higher level in the region of interest or activity, thus considerably reducing the computational cost. This method has been extensively used by Wieczorek and Dannenberg ${ }^{32}$ to demonstrate the stability and cooperative interactions due to $\mathrm{H}$ bonds of $\beta$ strands, $\alpha$ helices, and 310 helices of polyalanine.

In the present paper, a new version of MTA, viz, CGMTA is developed and applied within the DFT framework employing the B3LYP functional ${ }^{33}$ for geometry optimization. We first employ our strategy to simple systems such as $\alpha$-tocopherol and later extend it to more intricate molecules such as taxol and cyclodextrin containing several noncovalent interactions. The method is also applied to two conformations of polyglycines. Comparison of timings, electronic energies, and gradients with the actual computations is reported wherever possible. Distributed and parallel implementation of CG-MTA is also presented, which clearly brings out the ease with which grid-enabled ab initio computations could be achieved. For the sake of comparison, a few results at HF and MP2 levels of theory are also included. However, the methodology presented is general and may be employed at any level of theory.

\section{METHODOLOGY}

The technique involved in MTA is fundamentally that of a divide-and-conquer approach, wherein the parent system is broken down into a series of overlapping fragments. Instead
(A)

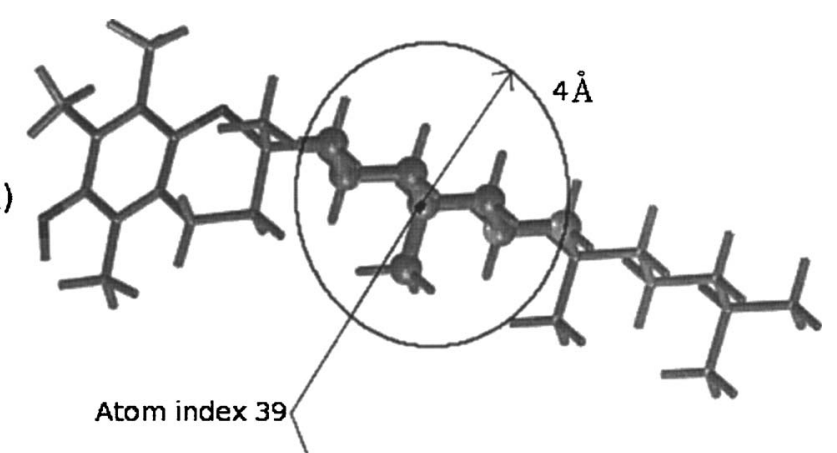

(B)

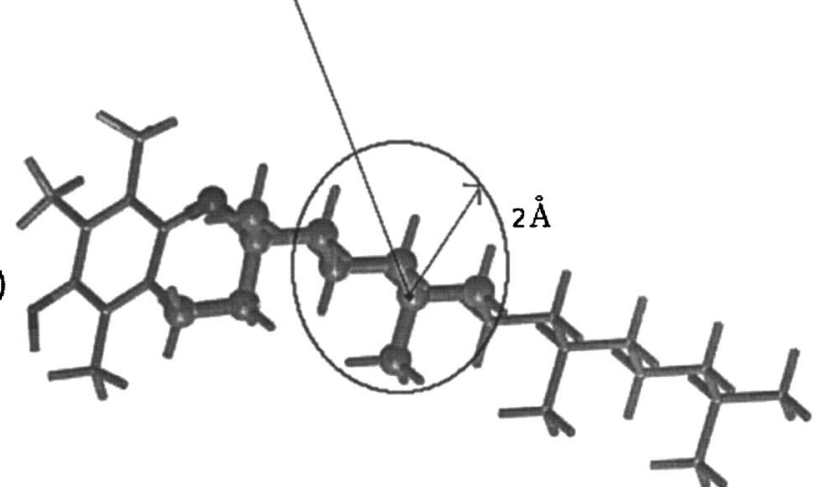

FIG. 1. The $R$ goodness of atom 39 in fragment $\mathrm{A}$ is $4 \AA$ while in fragment B it is $2 \AA$, and hence the atom 39 is best represented in fragment A with an $R$ goodness of $4 \AA$. The portion of the molecule that is a part of the molecule is displayed in a ball and stick model, while the rest of the portion is displayed as a stick model.

of performing a formidable computation on the supermolecule, only smaller subsystems (fragments) are subjected to computation. Our earlier work on MTA has largely concentrated on the estimation of one-electron properties, and its accuracy and efficiency have been demonstrated in the literature. ${ }^{11-15}$ For applying MTA to optimization of large molecules, ${ }^{14,15}$ the fragmentation scheme has been considerably modified for tracking the spatial nature of the molecule by placing spheres of appropriate dimension along the molecular skeleton to represent the initial fragments. These initial fragments are then merged according to their proximity and overlap and adhering to certain minimum and maximum sizes with connectivity constraints. Effective implementation of MTA for geometry optimization requires a quantitative definition of the quality of a given fragmentation scheme. This is done by defining the $R$ goodness of an atom $A_{i}$ in fragment $F_{i}$, which is taken to be the maximum radius of the sphere centered on $A_{i}$ such that all the atoms within this sphere are also included in $F_{i}$. As an example refer to Fig. 1, in which an atom marked 39 appears in two fragments, A and B (the highlighted portion of the molecular skeleton represents the atoms in the respective fragments). The maximum radius of the sphere that can be drawn from the nuclear position of atom 39 in fragment $A$ such that all the atoms that fall within this sphere are also a part of fragment A is $4 \AA$. Similarly, for fragment $\mathrm{B}$ this value is $2 \AA$. Now, the $R$ goodness of atom 39 is taken to be the maximum of these two, i.e., $4 \AA$ as in fragment A. Next, the minimum of $R$ goodness of all the atoms in the parent molecule is taken to be the $R$ goodness of a fragmentation scheme. A higher $R$ goodness with comparatively lower number of atoms per fragment is 


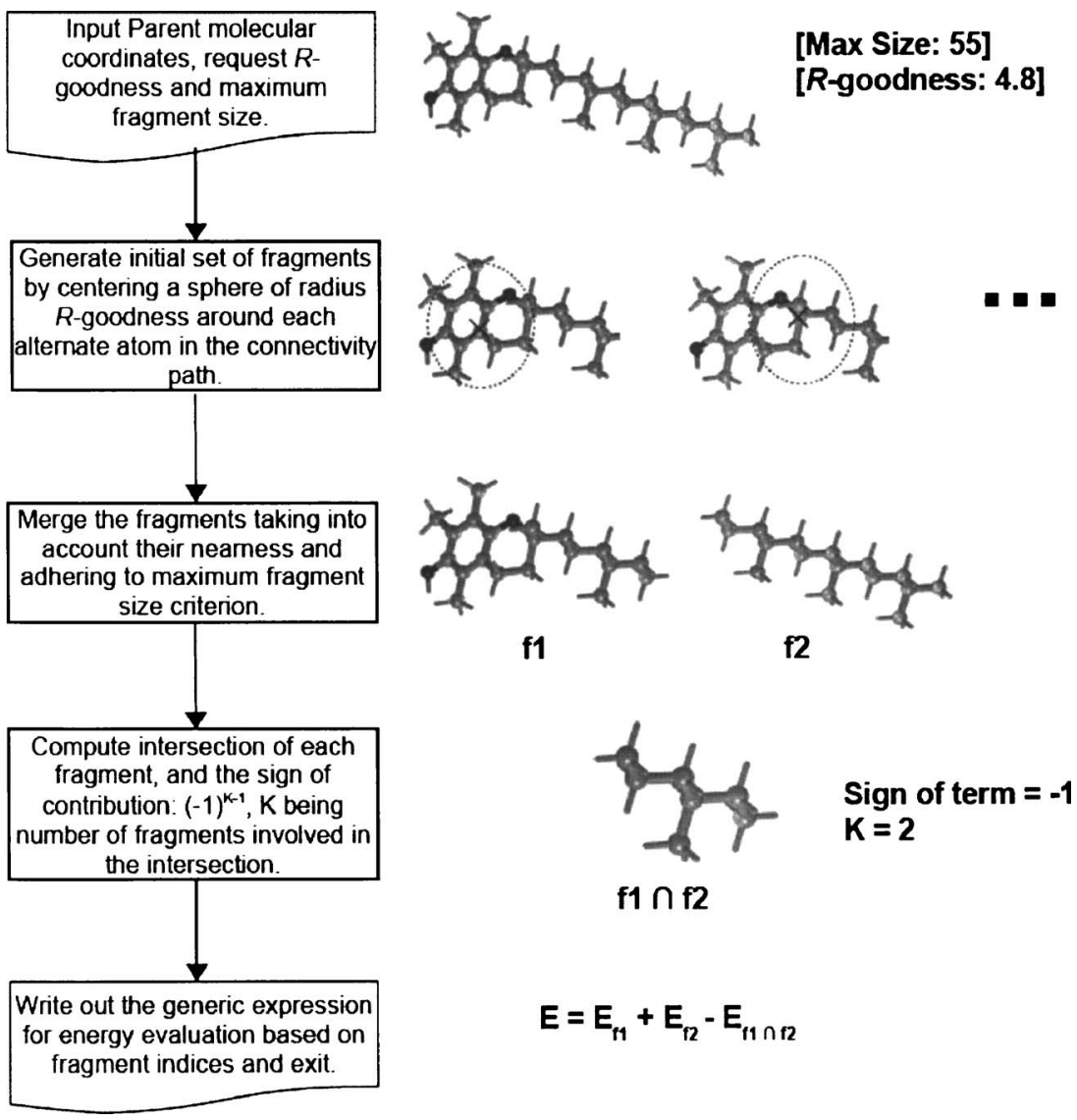

FIG. 2. A flow chart of automated fragmentation scheme, along with representative schematics of fragmentation process acting on $\alpha$-tocopherol.

considered to be a better scheme than the ones with lower $R$ goodness. ${ }^{14,15}$ The gist of the fragmentation method is a general and automated scheme that allows obtaining better estimates of the chemical environment of the supermolecule by appropriately adjusting the $R$-goodness value in the fragmentation procedure.

As the actual energy of the whole molecule is never evaluated throughout the minimization procedure, there must be some way to quantitatively assess the lowering of the energy of the whole system. For this purpose, we define fragment ensemble energy $\left(\Sigma E^{f i}\right)$, which is merely a summation of fragment energies as a parameter that mimics the lowering of total energy $(E)$ of the system quite well. ${ }^{14,15}$ It is very interesting to note that the variation in this parameter during the optimization cycles qualitatively reflects the changes in the total energy of the system. However, for a quick but yet quantitatively more accurate estimation of the total energy, the earlier MTA (Ref. 15) needs to be modified. This is achieved in the present work by obtaining a total energy estimate based on adjusting the cardinality (i.e., the number of elements in a set) of the fragments and their overlaps, hence the name CG-MTA. In the current context, the number of atoms and bonds refers to the cardinality of a fragment. From the set inclusion and exclusion principle, ${ }^{34}$ the cardinality of a union of $N$ sets can be derived from the summation of cardinalities of individual sets minus the cardinalities of the intersecting parts. In a similar spirit, the energy equation (as well as the equations for derivatives), as described below, is written in a manner that preserves the count of atoms and bonds in the parent system.
To illustrate this, we consider a molecule (say, $A$ ) whose molecular formula is $\mathrm{C}_{29} \mathrm{O}_{2} \mathrm{H}_{50}$ ( $\alpha$-tocopherol). The automated fragmentation process briefly described above is provided with coordinates of the parent molecule, the desired $R$-goodness value, and the maximum allowed fragment size. While $R$ goodness ensures that each atom has at least the goodness specified in the input, the maximum fragment size constraint ensures that the fragments do not cross a specified size limit (possibly restricted by the hardware resources available to handle the largest subsystem). For this example (Fig. 2), we choose an $R$-goodness value of 4.8 a.u. with a maximum size constraint of 55 atoms. Based on this minimal input the fragmentation process ${ }^{14,15}$ generates a set of main overlapping fragments, which in this case resulted into two fragments: $\mathrm{C}_{16} \mathrm{H}_{33}(f 1)$ and $\mathrm{C}_{20} \mathrm{O}_{2} \mathrm{H}_{31}(f 2)$. After this process is complete, the intersections are evaluated and recorded along with a sign to indicate additive or subtractive contribution to the energy (or gradient) expression. The overlapping fragment in this case turns out to be $\mathrm{C}_{7} \mathrm{H}_{14}(f 1 \cap f 2)$, which needs to be subtracted as it is a binary overlap. Dummy hydrogens are then added to satisfy the valencies of the cut bonds. Summing up, stoichiometrically the supermolecular formula could be constructed as [Fig. 3(A)]

$$
\mathrm{C}_{29} \mathrm{O}_{2} \mathrm{H}_{50} \Rightarrow \mathrm{C}_{16} \mathrm{H}_{33}+\mathrm{C}_{20} \mathrm{O}_{2} \mathrm{H}_{31}-\mathrm{C}_{7} \mathrm{H}_{14} \text {. }
$$

Using the above fragmentation pattern, the energy can now be estimated as

$$
E^{A}=E^{f 1}+E^{f 2}-E^{f 1 \cap f 2} .
$$




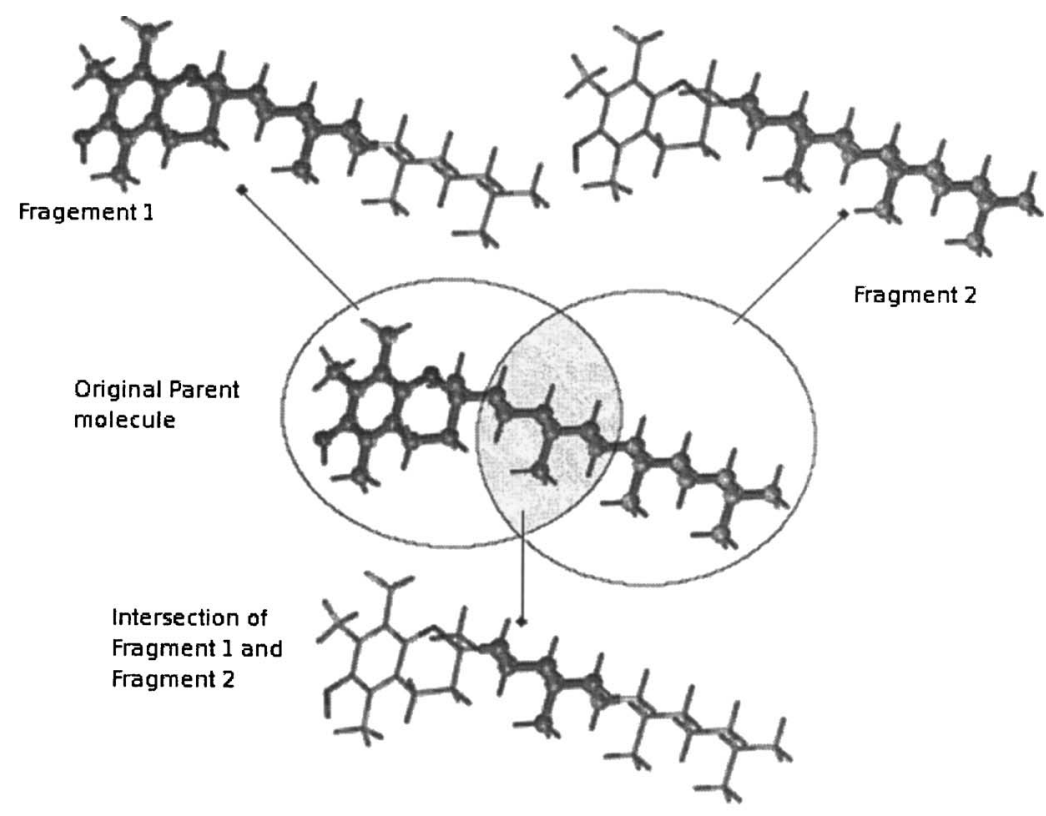

A

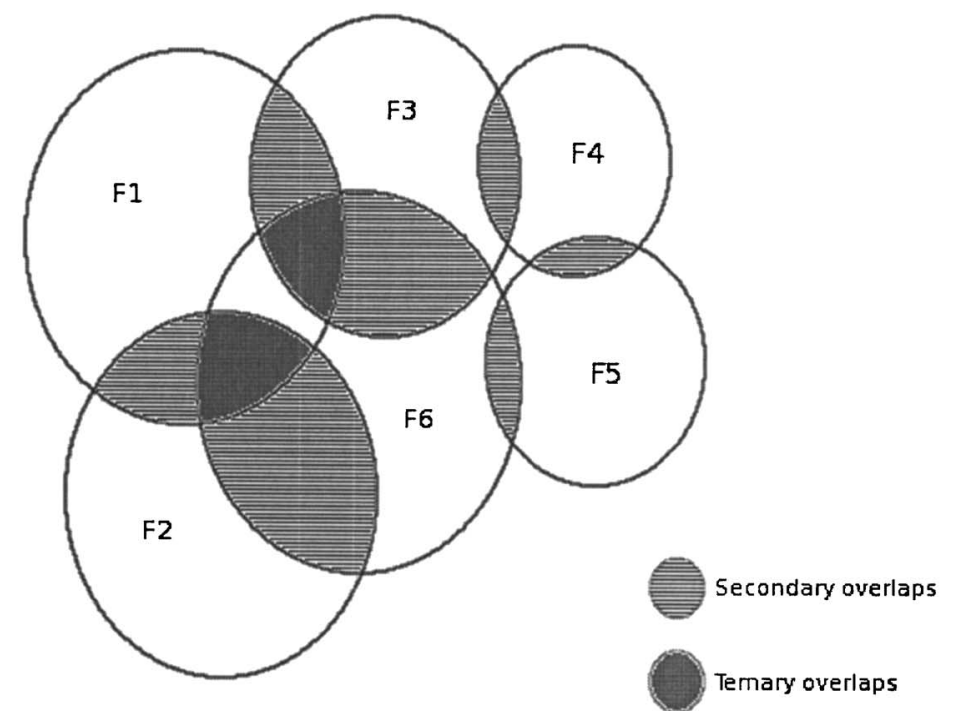

FIG. 3. A Venn diagrammatic representation of fragments and its overlaps. (A) Actual fragments of $\alpha$-tocopherol and the corresponding overlapping fragment. (B) Schematic representation of a general case with secondary and ternary overlapping fragments.
The above expression only holds true for fragments with binary overlaps. In general, for a nonlinear chemical system, it is but common to have ternary (or higher) overlaps. One such situation is schematically depicted using a Venn diagram in Fig. 3(B) with a total of six fragments, seven binaryand two ternary overlaps. The energy expression for this system could be written as

$$
\begin{aligned}
E^{A}= & \sum_{i=1}^{6} E^{f i}-E^{f 1 \cap f 2}-E^{f 1 \cap f 3}-E^{f 2 \cap f 6}-E^{f 3 \cap f 6}-E^{f 3 \cap f 4} \\
& -E^{f 4 \cap f 5}-E^{f 5 \cap f 6}-E^{f 1 \cap f 2 \cap f 6}+E^{f 1 \cap f 3 \cap f 6}
\end{aligned}
$$

Generalizing the above expression for $k$ fragments, one obtains

$$
E^{A}=\sum E^{f i}-\sum E^{f i \cap f j}+\cdots+(-1)^{k-1} \sum E^{f i \cap f j \cap \cdots \cap f k} .
$$

Using the above expression for energy, the gradients can now be estimated as

$$
\begin{aligned}
\frac{\partial E^{A}}{\partial X_{\mu}}= & \sum \frac{\partial E^{f i}}{\partial X_{\mu}^{f i}}-\sum \frac{\partial E^{f i \cap f j}}{\partial X_{\mu}^{f i \cap f j}} \\
& +\cdots+(-1)^{k-1} \sum \frac{\partial E^{f i \cap f j \cap \cdots \cap f k}}{\partial X_{\mu}^{f i \cap f j \cap \cdots \cap f k}} .
\end{aligned}
$$

Note that in the above equation, $X_{\mu}^{f i}$ refers to the nuclear coordinates of atom $\mu$ in fragment $f_{i}$, whereas $X_{\mu}^{f i \cap f j}$ refers to the coordinates of the overlap fragment $f_{i} \cap f_{j}$, etc. For computation of the Hessian matrix, a similar expression could be written as

$$
\begin{aligned}
\mathbf{H}_{a b}= & \sum \mathbf{H}_{a b}^{f i}-\sum \mathbf{H}_{a b}^{f i \cap f j} \\
& +\cdots+(-1)^{k-1} \sum \mathbf{H}_{a b}^{f i \cap f j \cap \cdots \cap f k} .
\end{aligned}
$$


It is to be noted that this method of energy evaluation is similar to the one proposed by Deev and Collins ${ }^{21}$ and Chen et $a .^{22}$ However, the energy (along with its first and second derivatives) evaluation scheme presented here is general and can be applied to any nonlinear system. CG-MTA also takes into account the effect of weak interactions (such as hydrogen bonding and $\mathrm{C}-H . . \pi$ interaction) by using $R$ goodness to quantify the goodness of a fragmentation scheme, which is evidently missing in earlier works. ${ }^{21,22}$ For systems with charge centers, the above expression still holds true. However, it is important to explicitly account for interactions between charge centers if these moieties are not included as a part of a single fragment. The automated fragmentation routine detects fragments with charge centers, and provides an option to add extra fragments centered at these places to cater to charged fragment-fragment interaction. Further, the expression in the form presented here could easily be translated into an efficient and automated computer code by cleverly employing a simple lexicographical combination generator (see, for instance, Ref. 35). Ideally, the cardinality expression once formed can be used throughout the optimization cycle. But it may also happen that during the optimization cycle, due to a drop in the $R$-goodness value, it would be required to refragment the parent system, in which case the cardinality expression is recalculated.

The automated fragmentation process generates fragment subsystems that have cut bonds. To satisfy their valencies hydrogen atoms are added. The positions of these atoms are generated with standard bond distances for the connected "real" atom positions. ${ }^{12}$ During an optimization cycle, these positions are recalculated based on the changed coordinates of the actual atoms. However, no effort is made to individually optimize the position of these dummy atoms as their effect is essentially canceled out by a similar dummy atom in the adjoining fragment.

The desirability of applying a parallel code for fragmentation-based methods has been noted earlier, ${ }^{17,21}$ but none have reported the implementation of actual test cases. In this paper, we use a parallelization strategy similar to our earlier work, ${ }^{15}$ with a few exceptions as noted below. In the default distributed mode of MTA run, the computational framework is arranged in the form of one originating client, and one or more compute nodes (also called compute servers) with possibly varying computing speeds and storage availability. The originating client does the job of fragmenting the supermolecule into small fragments and then scheduling the jobs on available compute nodes. The scheduling mechanism addresses important issues in a distributed network such as varying degree of computational power or the possibility of one or more nodes failing during job execution. If a tightly parallel system is available, then the distributed algorithm takes advantage of this and executes fragment jobs using GAMESS parallelization option on a virtual cluster (Fig. 4), thus allowing maximum utilization of computing resources. This is especially useful when the number of fragments is far less than the number of available nodes, in which case a set of tightly coupled nodes behave as a single compute server on which a GAMESS parallel fragment job is executed (see scheme 1 of Fig. 4). One of the crucial aspects

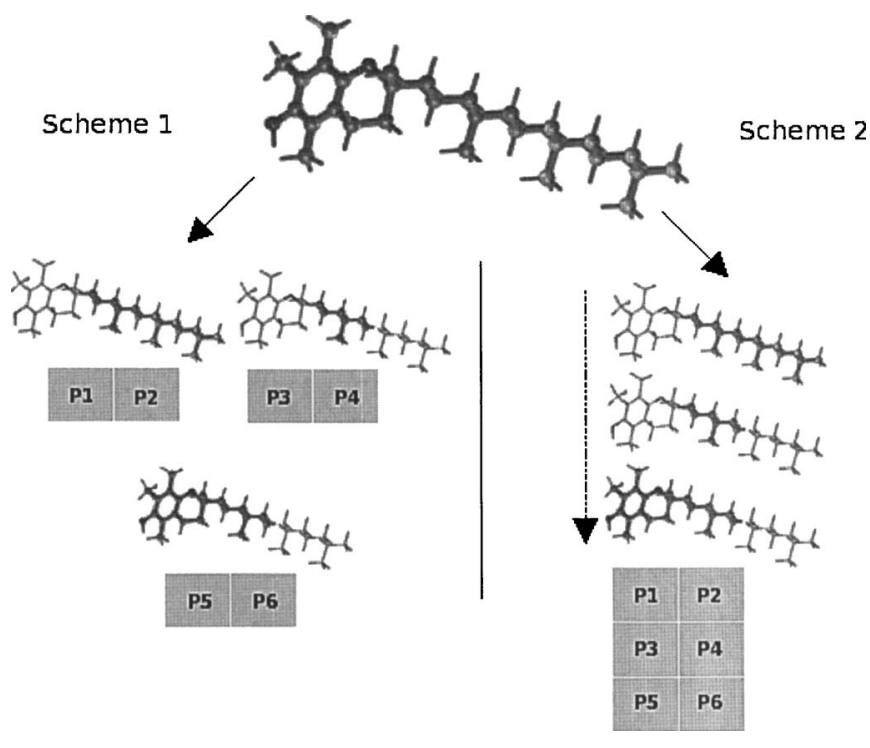

FIG. 4. Two different schemes of distributed CG-MTA run, for tightly coupled parallel architecture. One square is indicative of one compute node. Scheme 1 creates virtual clusters (two nodes each in the figure) and executes a number of fragment jobs in parallel. Scheme 2 executes one fragment at a time, but uses all the available processors (six in the figure) for each of these tasks.

of achieving scalability for a parallel algorithm is to evenly distribute the job on available compute nodes with proper synchronization. On a homogeneous setup of nodes, this is trivially achieved by evenly splitting the workload for an embarrassingly parallelizable algorithm such as CG-MTA. For a heterogeneous setup, wherein the computing powers of individual nodes differ and are not generally known a priori, a different strategy needs to be exploited. In its current form, the distributed version of CG-MTA algorithm performs automatic load balancing by lazy scheduling of the fragment selfconsistent-field (SCF) calculations on the compute nodes available in the network. ${ }^{14,15}$ This means that initially a set of $n$ fragment jobs of the total $M$ is sent to independent nodes. The node which finishes first is sent the next $(n+1)$ th fragment job, and so on. This late distribution of work among compute node allows for near optimum utilization of heterogeneous computing power. In essence, a faster node may handle computation of two fragments in, say, time $T$, whereas at the same time, a slower node may compute only a single fragment job. Another key aspect of a distributed setup is fault tolerance. In any given distributed setup, there is a chance that one or more nodes may fail because of some unknown reasons (power failure, network errors, etc.). A good algorithm should take care of such unpredictability and reconfigure itself at runtime so as to continue working without disruption of the ongoing process. The current implementation of CG-MTA takes into account such situations by performing a heartbeat operation, whereby periodic keepalive packets are sent from the originating client to all the nodes participating in a computational process (note that the participating nodes can also be dynamically added at runtime). In case a compute node stops responding to the periodic check it is kept in a list of inactive nodes, and the current running fragment job is transferred to other active nonbusy nodes. The nodes in the inactive list are checked 
TABLE I. Energy and gradient comparison at various stages of actual and CG-MTA-based optimization run for $\alpha$-tocopherol at B3LYP/STO-3G level of theory.

\begin{tabular}{|c|c|c|c|c|c|c|}
\hline \multirow[b]{2}{*}{ Iteration } & \multicolumn{2}{|c|}{ Energy (a.u.) } & \multicolumn{2}{|c|}{ Gradients (a.u.) $^{\mathrm{a}}$} & \multicolumn{2}{|c|}{ Time $(\min )^{\mathrm{b}}$} \\
\hline & CG-MTA & Actual & CG-MTA & Actual & CG-MTA & Actual \\
\hline 1 & -1269.17529 & -1269.17529 & $(0.03210,0.00777)$ & $(0.03210,0.007$ 77) & 12 & 20 \\
\hline 30 & -1269.19634 & -1269.19633 & $(0.00077,0.00021)$ & $(0.00079,0.00021)$ & 377 & 599 \\
\hline 60 & -1269.19658 & -1269.19658 & $(0.00032,0.00011)$ & $(0.00029,0.00009)$ & 739 & 1214 \\
\hline 90 & -1269.19665 & -1269.19665 & $(0.00009,0.00003)$ & $(0.00013,0.00004)$ & 1120 & 1815 \\
\hline
\end{tabular}

${ }^{\mathrm{a}}$ The values in parentheses indicate maximum and rms gradients, respectively.

${ }^{\mathrm{b}}$ Timings on four $2.8 \mathrm{GHz}$ PIV with 1 Gbytes RAM each.

upon periodically to access their availability and are pulled back into the active list if they are found to be functional. This mechanism of load distribution also allows for a clean way to dynamically attach/detach compute nodes as per requirements at runtime. Another scenario (scheme 2 in Fig. 4) is to run all the fragment jobs sequentially, but on a shared memory architecture using default GAMESS parallelization. This is useful if the fragments are large and individually take up a fair amount of computational resources. The distributed version implemented in the CG-MTA has some similarity to the way the DIVCON program is parallelized ${ }^{36}$ in that both of these algorithms use the "divide-and-conquer" technique. But the algorithm and implementation differs in a major way in that our implementation is targeted towards loosely coupled distributed machines with potentially varying degree of computational power and reliability. Also, because of the nature of CG-MTA there is no need for data replication which potentially allows for easy scaling over a large number of nodes.

This algorithm has been implemented in FORTRAN 90, and the code is linked to a locally modified version of GAMESS. ${ }^{37}$ Though the earlier discussion refers to the aspects of our implementation of CG-MTA algorithm in GAMESS, the algorithm per se is generic and can be easily implemented within the framework of any other $a b$ initio code such as GAUSSIAN 03. Also the parallelization strategy is general enough for easy integration with either a loosely distributed network of computers or tightly coupled shared memory systems.

\section{RESULTS AND DISCUSSION}

In order to assess the quality of energy and gradients during CG-MTA optimization, we first examine the performance of a complete optimization of a moderately large molecule, viz., $\alpha$-tocopherol $\left(\mathrm{C}_{29} \mathrm{O}_{2} \mathrm{H}_{50}\right)$ at minimal basis set, and compare it with the corresponding actual run. A near parallel path taken during the CG-MTA run, with a time saving of nearly $40 \%$ even for a small molecule like $\alpha$-tocopherol clearly is quite impressive (Table I). The maximum dynamic memory required during CG-MTA optimization was 50 Mbytes as compared to 66 Mbytes during actual calculation. This gap in memory requirements is expected to increase with increasing size of the molecule bringing out a different dimension of advantage in terms of considerably lesser memory requirements for CG-MTA runs. This is

TABLE II. Energy/gradient evaluation of some molecules at a random single point geometry on the optimization path using CG-MTA and its comparison with the actual computed energies and gradients (all values in a.u.).

\begin{tabular}{|c|c|c|c|c|}
\hline \multirow{2}{*}{$\begin{array}{l}\text { System (contractions) } \\
\text { (level/basis) }\end{array}$} & \multirow[b]{2}{*}{$\begin{array}{c}\text { Actual } \\
\left(G_{\max }, G_{\mathrm{rms}}\right)\end{array}$} & \multicolumn{2}{|c|}{ CG-MTA $\left(G_{\max }, G_{\mathrm{rms}}\right)^{\mathrm{b}}$} & \multirow[b]{2}{*}{$T_{r}^{\mathrm{c}}$} \\
\hline & & $4-5 \mathrm{~g} \AA$ & $2-3.5 \mathrm{~g} \AA$ & \\
\hline$\alpha$-tocopherol (889) & -1284.92615 & -1284.92612 & -1284.92609 & $4.2,5.2$ \\
\hline $\mathrm{B} 3 \mathrm{LYP} / 6-31++\mathrm{G}(d, p)$ & $(0.00049,0.00018)$ & $(0.00047,0.00010)$ & $(0.00039,0.00008)$ & \\
\hline Taxol (1185) & -2927.89660 & -2927.89652 & -2927.89739 & $2.3,3.1$ \\
\hline $\mathrm{B} 3 \mathrm{LYP} / 6-31 \mathrm{G}(d, p)$ & $(0.00304,0.00051)$ & $(0.00252,0.00043)$ & $(0.00799,0.00142)$ & \\
\hline$\gamma$-cyclodextrin $(1480)$ & -4883.54609 & -4883.54567 & -4883.53545 & $3.1,4.8$ \\
\hline $\mathrm{B} 3 \mathrm{LYP} / 6-31 \mathrm{G}(d, p)$ & $(0.00166,0.00042)$ & $(0.00150,0.00031)$ & $(0.00272,0.00047)$ & \\
\hline Extended polyglycine (1610) & -4406.50387 & -4406.50344 & -4406.50142 & $4.8,6.4$ \\
\hline $\mathrm{B} 3 \mathrm{LYP} / 6-31 \mathrm{G}(d, p)$ & $(0.00192,0.00047)$ & $(0.00167,0.00035)$ & $(0.00208,0.00041)$ & \\
\hline$\alpha$-helix polyglycine (1610) & -4406.58652 & -4406.57442 & -4406.53126 & $2.8,4.1$ \\
\hline $\mathrm{B} 3 \mathrm{LYP} / 6-31 \mathrm{G}(d, p)$ & $(0.04128,0.00436)$ & $(0.04246,0.00431)$ & $(0.04386,0.00431)$ & \\
\hline
\end{tabular}

${ }^{\mathrm{a}}$ The molecular formulae of the molecules are $\alpha$-tocopherol $\left(\mathrm{C}_{29} \mathrm{O}_{2} \mathrm{H}_{50}\right)$, taxol $\left(\mathrm{C}_{47} \mathrm{O}_{14} \mathrm{NH}_{51}\right), \gamma$-cyclodextrin $\left(\mathrm{C}_{48} \mathrm{O}_{40} \mathrm{H}_{80}\right)$, extended polyglycine $\left(\mathrm{C}_{43} \mathrm{O}_{21} \mathrm{~N}_{21} \mathrm{H}_{67}\right)$, and $\alpha$-helix polyglycine $\left(\mathrm{C}_{43} \mathrm{O}_{21} \mathrm{~N}_{21} \mathrm{H}_{67}\right)$.

${ }^{\mathrm{b}} G_{\mathrm{max}}$ and $G_{\mathrm{rms}}$ represent maximum and rms energy gradients in a.u., respectively.

${ }^{c}$ Ratio of time taken for actual single point run to that taken for CG-MTA run using a fragmentation scheme with goodness values between $4-5$ and $2-3.5 \AA$, respectively. 


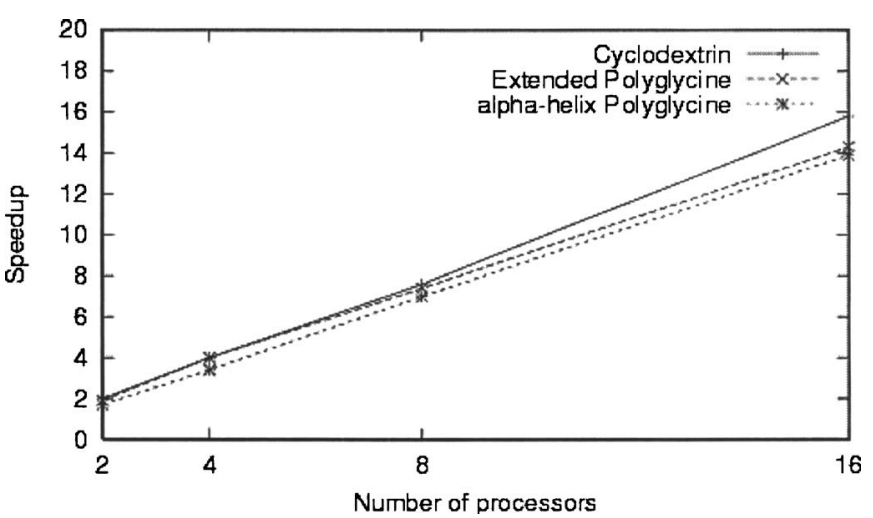

FIG. 5. Scaling of distributed CG-MTA code in terms of CPU time taken for $\gamma$-cyclodextrin, extended polyglycine, and $\alpha$-polyglycine [at B3LYP/6$31 \mathrm{G}(d, p)$ level of theory] runs on $2,4,8$, and 16 Pentium IV processors $(2.8$ $\mathrm{GHz}$ with 2 Gbyte RAM each). The speedup is calculated as ratio of clock time required on one processor to that on $N$ processors.

clearly evident from the fact that at the B3LYP/6-31+ $+\mathrm{G}(d, p)$ level for $\alpha$-tocopherol, the maximum dynamic requirements for actual computation is about 700 Mbytes. At the same time, a maximum of only 250 Mbytes of dynamic memory is required for CG-MTA run, a clear saving of about $65 \%$ of the total memory requirement.

A comparison of final geometry parameters obtained from CG-MTA run and actual calculation indicates a maximum deviation of $1.37 \times 10^{-4} \AA, 0.004^{\circ}$, and $0.149^{\circ}$ in bond lengths, bond angles, and dihedral angles, respectively. This brings out the accuracy of CG-MTA in predicting structures. The energy and gradient values obtained from CG-MTA also match extremely well with their actual counterparts at every stage of optimization (Table I).

To test the accuracy of energy and gradients for other levels of theory and with different $R$-goodness values, a series of tests were performed, some of which are summarized in Table II. It is observed that, on average, energy evaluated using CG-MTA is able to match the total energy correct to two to three significant places after the decimal point. Energy evaluation using fragmentation schemes with various $R$ goodness indicates that typically a goodness of $3-4 \AA$ is enough to obtain energy estimates correct to a few millihartrees. For systems that have large steric hindrance (such as $\alpha$-helix polyglycine), better $R$-goodness values (5-6 $\mathrm{A}$ ) are required to obtain good quantitative agreement with the actual energies.

Extending our method to a correlated level of theory, viz., MP2, we have also performed a test calculation on taxol at 6-31G $(d)$ and 6-31G $(d, p)$ levels of theory with the energy values being -2920.60893 and -2921.02403 a.u., respectively. Actual MP2/6-31G $(d)$ energy at the former geometry was -2920.60623 a.u., a difference of about 3.7 millihartree from the estimated energy. A similar actual calculation could not be performed at the 6-31G $(d, p)$ basis on the same hardware because of memory restriction, fortifying our earlier claim of enabling certain calculations otherwise impossible on the currently popular PC hardware.

In order to probe the suitability of CG-MTA for Hessianbased optimization of large molecules, a simple test calculation on $\alpha$-tocopherol at HF/STO-3G has been performed. The CG-MTA-based Hessian is computed from Eq. (6). The maximum error in the estimated Hessian was $9.8 \times 10^{-4}$ a.u. While the actual Hessian computation took around $140 \mathrm{~min}$, CG-MTA-based estimation took merely $50 \mathrm{~min}$ on a single Pentium IV with 2 Gbyte random access memory (RAM). The most striking advantage of CG-MTA was that the maximum amount of memory required was about $25 \%$ of the memory required to perform the full calculation. A Hessian thus obtained may be used during the optimization stages as an alternative to the estimated Hessian guess provided by $a b$ initio packages. Using the Hessian within CG-MTA, it also becomes easier to obtain the vibrational frequencies of a large molecule with comparatively less hardware resources. In fact, in the tests performed, we were able to obtain all the vibrational frequencies of $\alpha$-tocopherol at HF/STO-3G correct to all integer places.

A few tests were performed to demonstrate the scalability of distributed implementation (scheme 2 in Fig. 4) of CG-MTA algorithm (Fig. 5). From the tests performed on $\gamma$-cyclodextrin, $\alpha$-helix, and extended polyglycine at B3LYP/6-31G $(d, p)$ level on $2,4,8$, and 16 CPUs, a near-

TABLE III. Energy and gradients (both in a.u.) of initial and final molecular geometries from B3LYP CG-MTA run.

\begin{tabular}{|c|c|c|c|c|c|c|}
\hline \multirow[b]{2}{*}{$\begin{array}{c}\text { System } \\
\left(N_{a}, N_{c}, N_{f c}\right)^{\mathrm{a}}\end{array}$} & \multicolumn{3}{|c|}{ Initial geometry } & \multicolumn{3}{|c|}{ Final geometry } \\
\hline & Energy & $G_{\max }$ & $G_{\mathrm{rms}}$ & Energy & $G_{\max }$ & $G_{\mathrm{rms}}$ \\
\hline $\begin{array}{l}\alpha \text {-tocopherol } \\
(81889500)\end{array}$ & -1284.92562 & 0.00679 & 0.00076 & -1284.92612 & 0.00047 & 0.00010 \\
\hline $\begin{array}{c}\text { Taxol } \\
\text { (113 1185 715) }\end{array}$ & -2927.83639 & 0.6280 & 0.01320 & -2927.89756 & 0.00264 & 0.00070 \\
\hline $\begin{array}{c}\gamma \text {-cyclodextrin } \\
(1681480485)\end{array}$ & -4883.34370 & 0.17812 & 0.01891 & -4883.55609 & 0.00047 & 0.00014 \\
\hline $\begin{array}{l}\text { Extended polyglycine } \\
\quad(1521610485)\end{array}$ & -4606.41430 & 0.03260 & 0.01490 & -4406.50737 & 0.00086 & 0.00025 \\
\hline $\begin{array}{l}\alpha \text {-helix polyglycine } \\
\quad(1521610485)\end{array}$ & -4606.45450 & 0.04740 & 0.01240 & -4406.57792 & 0.00293 & 0.00043 \\
\hline
\end{tabular}


(A)

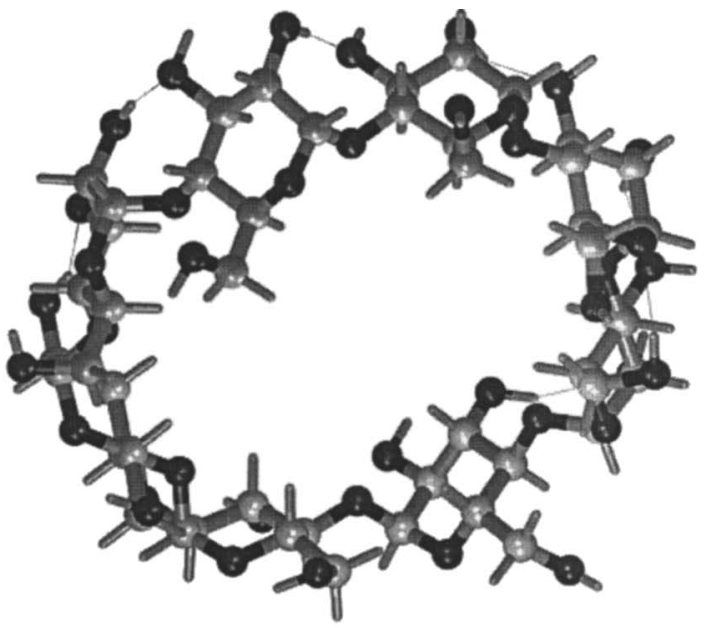

(B)

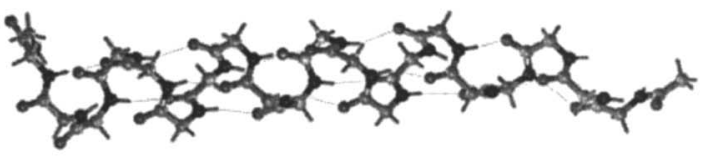

(C)

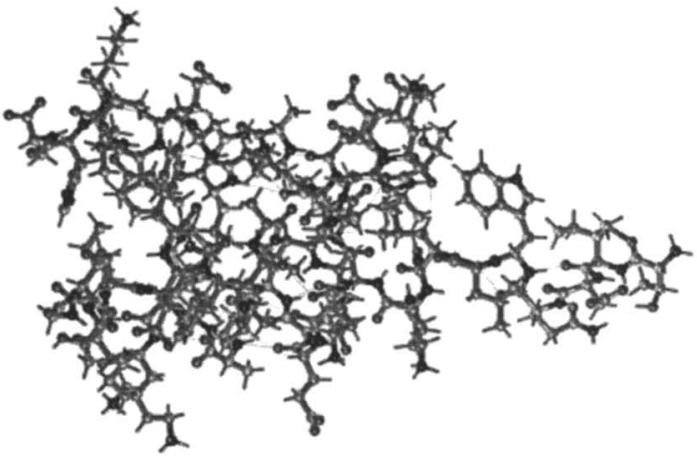

(D)

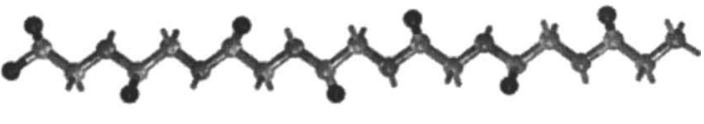

FIG. 6. Final CG-MTA optimized geometries. (A) $\gamma$-cyclodextrin (B) $\alpha$-helix glycine (C) 1 prb albumin binding protein. (D) A system with two charge centers $\mathrm{NH}_{3}^{+}\left(\mathrm{CH}_{2} \mathrm{CONH}\right)_{6} \mathrm{CH}_{2} \mathrm{COO}^{-}$. See text for details.

linear scaling is observed. Further, the time required for communication of compute servers with the originating nodes is minimal (not exceeding $1 \mathrm{~min}$ in general for all the cases reported) as compared to typical times of 10-60 min required for fragment calculation. Thus, the distributed implementation of CG-MTA could even be used over a very loosely connected network without drastically affecting the overall performance.

Initial and final energies as well as gradients of a few geometries (Table III and Fig. 6) are indicative of the strength of using CG-MTA-based structure determination even for intricate three-dimensional (3D) structures like $\gamma$-cyclodextrin and $\alpha$-helical glycine. A few of the optimization runs reported here have been performed on a 16 processor Power-5 machine, taking a maximum CPU time of 4 days per job in shared mode for full geometry convergence (all gradients numerically less than $5 \times 10^{-4}$ a.u.). Not all geometries reported here are run till full convergence, but are run sufficiently to demonstrate the use of CG-MTA for large scale calculations.

To bring out the scalability of CG-MTA algorithm for
TABLE IV. Partial geometry optimization of 851 atom protein (PDB ID: 1prb) at HF/3-21G, followed by a few steps at B3LYP/3-21G with 4635 basis functions.

\begin{tabular}{cccc}
\hline \hline Iteration & Energy (a.u.) & Gradients (a.u.) $)^{\mathrm{a}}$ & Time (min.) \\
\hline \multicolumn{4}{c}{ HF/3-21G } \\
1 & -20162.81113 & $(0.14024,0.001143)$ & $633^{\mathrm{b}}$ \\
30 & -20163.83649 & $(0.01406,0.00145)$ & $11422^{\mathrm{b}}$ \\
50 & -20164.04792 & $(0.00932,0.00130)$ & $17653^{\mathrm{b}}$ \\
\multicolumn{4}{c}{ B3LYP/3-21G } \\
1 & -20275.78706 & $(0.04604,0.00924)$ & $342^{\mathrm{c}}$ \\
3 & -20275.93497 & $(0.03661,0.00289)$ & $1000^{\mathrm{c}}$ \\
6 & -20276.01131 & $(0.01171,0.00117)$ & $2050^{\mathrm{c}}$ \\
\hline \hline
\end{tabular}

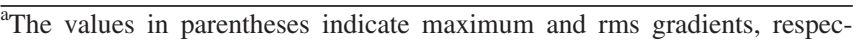
tively.

${ }^{\mathrm{b}}$ Timings on one $2.8 \mathrm{GHz}$ PIV with 1 Gbyte RAM.

'Timings on $162.8 \mathrm{GHz}$ PIV with 1 Gbyte RAM.

larger systems, it was applied to an albumin binding protein [Protein Data Bank (PDB) ID: 1prb, Fig. 6(C)] with 4635 basis functions at HF/3-21G level of theory. A fragmentation scheme with $R$-goodness value of $3.0 \AA$ and 15 main fragments were used for this calculation. The largest fragment had 628 basis functions, i.e., about $1 / 7$ of that for the complete molecule. In terms of memory requirements this translates into about 2 Gbytes for the whole molecule as compared to a maximum of merely 400 Mbytes for CG-MTA calculation. Hence, the actual calculation was not at all possible, whereas the CG-MTA job ran effortlessly on a single Pentium IV with 1 Gbyte RAM (Table IV). The final geometry obtained from HF run, was later subjected to a few runs at B3LYP level to demonstrate the ability of the algorithm to handle such systems. The systematic lowering of energy and gradients in Table IV clearly brings out the suitability of CG-MTA for handling large systems.

Though the current algorithm is not specifically designed to take into account the systems with one or more charge centers, we demonstrate with a small test calculation on $\mathrm{NH}_{3}^{+}\left(\mathrm{CH}_{2} \mathrm{CONH}\right)_{6} \mathrm{CH}_{2} \mathrm{COO}^{-}$[Table V, Fig. 6(D)] how such systems could be handled within CG-MTA. It is to be noted here that while performing SCF of fragments, appropriate charges $(+1$ and -1$)$ are added to the fragments which have charge imbalance. An initial test run without adding the fragment-fragment interaction results in a geometry which, when compared with actual calculation, indicates a maximum deviation of $2.6 \times 10^{-3} \AA, 0.35^{\circ}$, and $0.09^{\circ}$ in bond lengths, bond angles, and dihedral angles, respectively. However, the energy difference between the estimated CG-MTA and the actual calculation turns out to be quite large at $16.6 \mathrm{kcal} \mathrm{mol}^{-1}$. To account for this, an extra fragment is added (as indicated in Sec. II), with addition of the following two-body correction term in the energy (and similar for gradient) expression:

$$
\Delta^{2} E=+E_{i j}-E_{i}-E_{j} .
$$

The above correction is similar to the one proposed by Deev and Collins ${ }^{21}$ and Chen et al. ${ }^{22}$ However, instead of calculating the $E_{i j}$ from scratch, the patched density matrix ${ }^{38}$ obtained from calculations of $E_{i}$ and $E_{j}$ is provided for faster 
TABLE V. Energy and gradient comparison at various stages of actual and CG-MTA-based optimization run for a zwitterionic system $\left(\mathrm{NH}_{3}^{+}\left(\mathrm{CH}_{2} \mathrm{CONH}\right)_{6} \mathrm{CH}_{2} \mathrm{COO}^{-}\right)$at $\mathrm{HF} / 6-31 \mathrm{G}(d, p)$ level of theory.

\begin{tabular}{|c|c|c|c|c|}
\hline \multirow[b]{2}{*}{ Iteration } & \multicolumn{2}{|c|}{ Energy (a.u.) } & \multicolumn{2}{|c|}{ Gradients (a.u. $)^{\mathrm{a}}$} \\
\hline & CG-MTA & Actual & CG-MTA & Actual \\
\hline 1 & -1523.61116 & -1523.63839 & $(0.02848,0.00899)$ & $(0.02783,0.00881)$ \\
\hline 40 & $\begin{array}{c}-1523.62015 \\
{[-1523.64661]^{\mathrm{b}}}\end{array}$ & -1523.64668 & $(0.00041,0.00011)$ & $(0.00034,0.00008)$ \\
\hline
\end{tabular}

${ }^{a}$ The values in parentheses indicate maximum and rms gradients, respectively.

${ }^{\mathrm{b}}$ Actual single point energy at this geometry.

convergence of SCF. Using this corrected term, the estimated energy at the final geometry is -1523.64281 a.u., which is in error by only about $2.4 \mathrm{kcal} \mathrm{mol}^{-1}$ as against around $16.6 \mathrm{kcal} \mathrm{mol}^{-1}$ when disconnected charged moieties are not considered as an extra fragment.

It is known that in vacuum a system like the one mentioned in the above model calculation will fold from two ends with charge centers, possibly resulting into a proton transfer. However, as the optimizer gets stuck in a local minima such a phenomenon is not observed in our test calculation as well as the actual one.

To check the applicability of CG-MTA to such situations, we took yet another model system $\left(\mathrm{NH}_{3}^{+}\left(\mathrm{CH}_{2}\right)_{19} \mathrm{COO}^{-}\right.$, Fig. 7) with a slightly bent conformer and ran an AM1 calculation using CG-MTA for estimating energy and gradients, along with a parallel actual computation. The AM1 (Table VI) and later a calculation at HF/6 $-31 \mathrm{G}(d)$ both resulted in a loop structure (Fig. 7) in actual as well as CG-MTA calculations. This provides a clear indication of CG-MTA-based optimization working in line with the actual calculation even when charge centers are present. Further, it is well known ${ }^{39}$ in the literature that ab initio calculations using flexible basis sets lead to the result that zwitterionic form is not energy minimum, to which our result confirms. An extension of the current approach to continuum models thus seems worthwhile and will be followed up in future work. It will be of interest to check the stability of the
(A)

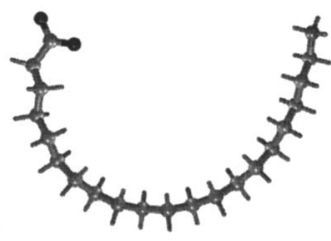

(B)

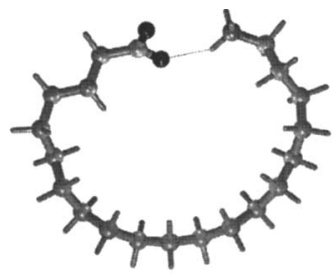

(C)

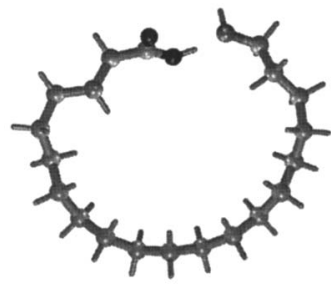

(B)

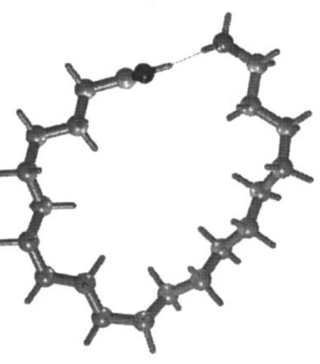

FIG. 7. Changes in geometry of $\mathrm{NH}_{3}^{+}\left(\mathrm{CH}_{2}\right)_{19} \mathrm{COO}^{-}$during CG-MTA optimization at AM1 level of theory. See text for details. zwitterionic form vis-a-vis that of the charge neutral system using such a treatment.

\section{CONCLUDING REMARKS}

In this paper, a general linear-scaling scheme for estimating energy and its derivatives for large molecules at $a b$ initio level by ensuring correct atom and bond cardinality of the supermolecular fragments is proposed. This method, termed as cardinality-guided molecular tailoring approach (CG-MTA), is applied for geometry optimization of a variety of molecules with varying degrees of complexity. Analysis of energy, gradients, and Hessian obtained from CG-MTA shows excellent agreement with the corresponding actual calculations. Further, the CG-MTA geometry optimization path is seen to closely mimic the one followed by actual calculation. This brings out the potential of CG-MTA for reliable optimization of large molecules at $a b$ initio level of theory. Molecules such as $\gamma$-cyclodextrin, polyglycine, and $1 \mathrm{prb}$, which are otherwise very difficult to optimize on a PC-class machine, could easily be handled using CG-MTA. Apart from the evident time advantage, substantial savings in terms of memory requirements allow otherwise impossible calculations to be performed on commodity PC hardware.

An initial attempt towards obtaining Hessian of energy using CG-MTA indicates generally a good agreement with the actual calculation, paving the way towards applying Hessian-based optimization as well as vibrational frequency calculations of large molecules in the future. The other advantage of the present algorithm is that it is relatively easy to run it over a distributed computing framework, utilizing the computing power as and when needed for handling large chemical systems. The nature of the algorithm is such that the communication time between the originating and the compute nodes is minimal, and hence even a simple ethernet network will not affect the overall performance of the distributed code. Though most of the optimization calculations performed by CG-MTA calculation have taken the maximum gradient norm below $5.0 \times 10^{-4}$, it may be noted that values of $1.0 \times 10^{-3}$ a.u. are currently an accepted norm for such large molecular systems. ${ }^{40}$ In the end, CG-MTA is not specifically developed for handling systems with many charge centers, but we have discussed a scheme in which we are able to obtain sufficiently good geometries in comparison to the actual run.

Encouraged by the CG-MTA geometry optimization results reported in the present work, we envisage applications 
TABLE VI. Energy and gradient comparison at various stages of actual and CG-MTA-based optimization run for a zwitterionic system $\mathrm{NH}_{3}^{+}\left(\mathrm{CH}_{2}\right)_{19} \mathrm{COO}^{-}$at $\mathrm{AM} 1$ and $\mathrm{HF} / 6-31 \mathrm{G}(d)$ (at a later geometry) level of theory.

\begin{tabular}{|c|c|c|c|c|}
\hline \multirow[b]{2}{*}{ Iteration } & \multicolumn{2}{|c|}{ Energy (a.u.) } & \multicolumn{2}{|c|}{ Gradients (a.u.) $^{\mathrm{a}}$} \\
\hline & CG-MTA & Actual & CG-MTA & Actual \\
\hline 1 & -146.02325 & -146.02332 & $(0.00688,0.00097)$ & $(0.00681,0.00096)$ \\
\hline 190 & -146.16107 & -146.16389 & $(0.02864,0.00558)$ & $(0.02723,0.00441)$ \\
\hline $200^{\mathrm{b}}$ & -146.19321 & -146.19295 & $(0.01056,0.00225)$ & $(0.01372,0.00248)$ \\
\hline \multirow[t]{2}{*}{530} & -146.20966 & -146.20961 & $(0.00009,0.00003)$ & $(0.00009,0.00003)$ \\
\hline & $\mathrm{HF} / 6-31 \mathrm{G}(d)^{\mathrm{c}}$ & & & \\
\hline 1 & -985.29059 & -985.29060 & $(0.07788,0.01510)$ & $(0.07791,0.01510)$ \\
\hline 20 & -985.37442 & -985.37442 & $(0.00702,0.00176)$ & $(0.00703,0.00176)$ \\
\hline $24^{\mathrm{b}}$ & -985.40279 & -985.40279 & $(0.02767,0.00334)$ & $(0.02786,0.00397)$ \\
\hline 120 & -985.44556 & -985.44542 & $(0.00089,0.00037)$ & $(0.00095,0.00084)$ \\
\hline
\end{tabular}

${ }^{\mathrm{a}}$ The values in parentheses indicate maximum and rms gradients, respectively.

${ }^{\mathrm{b}} \mathrm{A}$ proton transfer is seen around this geometry.

${ }^{\mathrm{c}}$ Calculation starting with an intermediate AM1 geometry employing ab initio MTA.

of it to full ab initio level quantum chemical treatment of large molecular systems and clusters. In particular, we foresee large potential for applying our methodology to the implementation of large scale and scalable ab initio molecular dynamics simulations. This opens up new avenues for addressing yet unsolved problems such as folding of biomolecules, motifs of interacting molecules in molecular crystals, etc. These investigations are actively being pursued in our laboratory.

\section{ACKNOWLEDGMENTS}

The computational facility from the Center for Development of Advanced Computing (C-DAC), Pune is gratefully acknowledged. The authors thank C-DAC, UGC (CAS program to the University of Pune) and the Council of Scientific and Industrial Research (CSIR), New Delhi for financial assistance. Computational resources have also been generously made available through the UPE program of the UGC to the University of Hyderabad for which the authors are grateful.

${ }^{1}$ G. E. Moore, Electronics 8, 38 (1965).

${ }^{2}$ A. Szabo and N.S. Ostlund, Morden Quantum Chemistry (McGraw-Hill, New York, 1989).

${ }^{3}$ W. J. Hehre, L. Random, and P. V. R. Schleyer, Ab Initio Molecular Orbital Theory (Wiley, New York, 1986).

${ }^{4}$ J. Almlöf, K. Faegri, and K. Krosell, J. Comput. Chem. 3, 385 (1982).

${ }^{5}$ D. L. Strout and G. E. Scuseria, J. Chem. Phys. 102, 8448 (1993).

${ }^{6}$ H. F. Schaefer III and C. J. Barden, Pure Appl. Chem. 72, 1405 (2000).

${ }^{7}$ Q. Zhao and W. Yang, J. Chem. Phys. 102, 9598 (1995).

${ }^{8}$ W. Yang and T. S. Lee, J. Chem. Phys. 103, 5674 (1995).

${ }^{9}$ W. Yang, Phys. Rev. Lett. 66, 1438 (1991).

${ }^{10}$ X. P. Li, R. W. Nunes, and D. Vanderbilt, Phys. Rev. B 47, 10891 (1993).

${ }^{11}$ S. R. Gadre, R. N. Shirsat, and A. C. Limaye, J. Phys. Chem. 98, 9165 (1994).

${ }^{12}$ K. Babu and S.R. Gadre, J. Comput. Chem. 24, 484 (2003).

${ }^{13}$ K. Babu, V. Ganesh, S. R. Gadre, and N. E. Ghermani, Theor. Chem. Acc. 111, 255 (2004).

${ }^{14}$ S. R. Gadre, K. Babu, and V. Ganesh, in Recent Trends in Practice and Theory of Information Technology: Proceedings of NRB Seminar, edited by S. N. Maheshwari (Viva Books, New Delhi, 2005).

${ }^{15}$ S. R. Gadre and V. Ganesh, J. Theor. Comput. Chem. (in press).
${ }^{16}$ T. E. Exner and P.G. Mezey, J. Phys. Chem. A 108, 4301 (2004).

${ }^{17}$ W. Li and S. Li, J. Chem. Phys. 122, 194109 (2005).

${ }^{18} \mathrm{~S}$. Li, W. Li, and T. Fang, J. Am. Chem. Soc. 127, 7215 (2005).

${ }^{19}$ T. Nakano, T. Kaminuma, T. Sato, Y. Akiyama, M. Uebayasi, and K. Kitaura, Chem. Phys. Lett. 318, 614 (2000).

${ }^{20}$ K. Kitaura, S. Sugiki, T. Nakano, Y. Akiyama, and M. Uebayasi, Chem. Phys. Lett. 336, 163 (2001).

${ }^{21}$ V. Deev and M. A. Collins, J. Chem. Phys. 122, 154102 (2005).

${ }^{22}$ X. Chen, Y. Zhang, and J. Z. H. Zhang, J. Chem. Phys. 122, 184 (2005).

${ }^{23}$ C. V. Alsenoy, Y. Ching-Hsing, A. Peters, J.M. L. Martin, and L. Schafer, J. Phys. Chem. A 102, 2246 (1998).

${ }^{24}$ T. Ijegami, T. Ishida, D. G. Fedorov, K. Kitaura, Y. Inadomi, H. Umeda, M. Yokokawa, and S. Sekiguchi, Proceedings of Supercomputing, 2005 (IEEE Computer Society, Seattle, WA, 2005), p. 10.

${ }^{25}$ D. G. Fedorov and K. Kitaura, J. Chem. Phys. 121, 2483 (2004).

${ }^{26}$ K. Fukuzawa, K. Kitaura, M. Uebayasi, K. Nakata, T. Kaminuma, and T. Nakano, J. Comput. Chem. 26, 1 (2005).

${ }^{27}$ D. G. Fedorov, R. M. Olson, K. Kitaura, M. S. Gordon, and S. Koseki, J. Comput. Chem. 25, 872 (2004).

${ }^{28}$ Y. Komeiji, T. Nakano, K. Fukuzawa, Y. Ueno, Y. Inadomi, T. Nemoto, M. Uebayasi, D. G. Fedorov, and K. Kitaura, Chem. Phys. Lett. 372, 342 (2003).

${ }^{29}$ M. J. Frisch, G. W. Trucks, H. B. Schlegel, et al. Gaussian 03, Revision C.02, Gaussian, Inc., Wallingford, CT, 2004.

${ }^{30}$ T. Vreven, K. Morokuma, O. Frakas, H. B. Schlegel, and M.J. Frisch, J. Comput. Chem. 24, 760 (2003).

${ }^{31}$ K. S. Byun, K. Morokuma, and M.J. Frisch, J. Mol. Struct.: THEOCHEM 462, 1 (1999).

${ }^{32}$ R. Wieczorek and J. J. Dannenberg, J. Am. Chem. Soc. 126, 14198 (2004).

${ }^{33}$ C. Lee, W. Yang, and R. G. Parr, Phys. Rev. B 37, 785 (1988).

${ }^{34}$ C. L. Liu, Elements of Discrete Mathematics (McGraw-Hill, New York, 1985).

${ }^{35}$ D. Knuth, Art of Computer Programming (Addison-Wesley, Reading, MA, 2005), Vol. 4, Fascicle 2/3.

${ }^{36}$ J. J. Vincent, S. L. Dixon, and K. M. Merz, Theor. Chem. Acc. 99, 220 (1998).

${ }^{37}$ M. S. Gordon, GAMESS package, 2003 (URL http:// www.msg.ameslab.gov/GAMESS/GAMESS.html).

${ }^{38}$ A. D. Kulkarni, V. Ganesh, and S. R. Gadre, J. Chem. Phys. 121, 5043 (2004).

${ }^{39}$ F. R. Tortonda, J. Pascual-Ahuir, E. Silla, and I. Tuñon, Theor. Chem. Acc. 99, 220 (1998).

${ }^{40}$ F. L. Gervasio, P. Carloni, and M. Parrinello, Phys. Rev. Lett. 89, 108102 (2002). 